\title{
Correction to: UV photometry of spotted stars in the horizontal branch of the globular cluster NGC 2808 using AstroSat
}

\author{
DEEPTHI S. PRABHU ${ }^{1,2, *}$, ANNAPURNI SUBRAMANIAM ${ }^{1}$ and SNEHALATA SAHU ${ }^{1}$ \\ ${ }^{1}$ Indian Institute of Astrophysics, Bangalore 560 034, India. \\ ${ }^{2}$ Pondicherry University, R.V. Nagar, Kalapet 605 014, India. \\ *Corresponding Author. E-mail: deepthi.prabhu@iiap.res.in
}

\section{Correction to: J Astrophys Astron}

https://doi.org/10.1007/s12036-020-09679-y

This is an erratum to the paper 'UV photometry of spotted stars in the horizontal branch of the globular cluster NGC 2808 using AstroSat' (J. Astrophys. Astron., 42, 66 2021). In the originally published version of this manuscript, one of the references was incorrectly typeset.
The incorrect reference was Prabhu D. S., Subramaniam A., Sahu S. 2021, J. Astrophys. Astr., 42, https://doi.org/10.1007/s12036-020-09679-y.

The correct reference is Prabhu D. S., Subramaniam A., Sahu S. 2021, Astrophys. J., 908, 66.

The original article has now been corrected. 